\title{
Dressing Based on Multilayer Nonwoven Materials with the Complex of Protective Properties
}

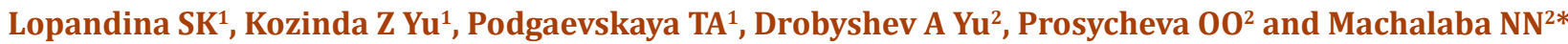 \\ ${ }^{1}$ Central Research Institute for Garment Industry, Russia \\ ${ }^{2}$ Medicine and Dentistry, Russia
}

*Corresponding author: Machalaba NN, Yevdokimov Moscow State University for Medicine and Dentistry, Russia

submission: 海 November 15, 2018; Published: 㭗 January 09, 2019

\section{Introduction}

Dressing made of materials that have both protective, antimicrobial and sorption properties are most effective in the treatment of postoperative wounds in surgical practice. The developed dressing material consists of fiber components of various chemical nature with one of the layers being treated with antimicrobial drugs [1].

Optimal ratios of raw components and structural parameters of multilayer nonwoven materials were developed based on the results of the research of the kinetics of sorption and desorption of liquid environments imitating exudate, as well as the diffusion of antimicrobial drugs from one structural component of the material to others and into the surrounding space [2]. It was established that the main impact on the absorbing ability of a multilayer nonwoven material is made by the raw material composition of the inner layer. To a lesser degree - the thickness of the material, its surface density and the number of layers [3-6].

There are two stages of the treatment of postoperative wounds, in particular, in oral and maxillofacial surgery: the first (1-5 day) with intensive exudate secretion and the second one (6-9 day) - with moderate exudate secretion. Based on this, two types of nonwoven material (type A, type B), which differ from each other by their surface density, thickness, number of layers and the raw material composition of the inner layer, were developed.

Table 1: Multilayer nonwoven materials for the first and second stages of treatment.

\begin{tabular}{|c|c|c|c|c|}
\hline Stages of Treatment of Postoperative Wounds & Surface Density, G/ $\mathbf{M}^{\mathbf{2}}$ & Number of Layers & Thickness, $\mathbf{M m}$ & $\mathbf{A b s o r b i n g ~ A b i l i t y , ~} \mathbf{M g} / \mathbf{C m}{ }^{\mathbf{2}}$ \\
\hline The first stage of treatment (1-5 day) & 500 & 4 & 20 & 700 \\
\hline The second stage of treatment (6-9 day) & 300 & 3 & 10 & 400 \\
\hline
\end{tabular}

The uniqueness of the developed material for dressings lies in its multilayer structure. As a layer that fits the wound surface a is canvas [7], made of hygroscopic fiber with antimicrobial treatment used. As the outer layer which retains absorbed exudate a canvas, made of a mixture of fibers, including a fiber with minimal hygroscopicity, is used. Characteristics of multilayer nonwoven materials are presented in Table 1.

Besides, dressing the multilayer nonwoven fabric of which lies between the outer layer of a hydrojet nonwoven material and the outer layer of a spunbond nonwoven material that are bonded by ultrasonic welding, was developed [8]. In this case, the outer layer is subjected to antimicrobial treatment. The main indicator characterizing the effectiveness of the dressing, along with its absorbency, is antimicrobial activity which depends on the chemical nature of the drug, its concentration in the material, diffusion into various layers of the material and into the wound cavity [9].

According to the Medical and Technical Requirements and Guidelines for the laboratory evaluation of the antimicrobial activity of textile materials containing antimicrobial drugs, a biologically active (antimicrobial) material is considered to be a material that, when tested in vitro, provides a growth inhibition zone for test microorganisms of at least $4 \mathrm{~mm}$ [10].
Drugs for antimicrobial treatment were selected on the basis of the results of microbiological research of canvases carried out in the laboratory of the Molecular and Biological Research of the National Institute for Microbiological Research and the Department of Microbiology of the Moscow State University of Medicine and Technology under the direction of Dr. med. Professor Tsarev V.N.

Microbiological tests of fibrous canvases treated with drugs of various classes allowed for use in medical practice (furan compounds, antibiotics, sulfonamides, quaternary ammonium compounds, chlorhexidine digluconate) showed that all of them have high antimicrobial activity, 2-3 times higher than the required value (4mm) [11]. Cefazolin and chlorhexidine digluconate in various concentrations were chosen for the treatment of fibrous canvases under production conditions.

Research conducted on models of wound surfaces showed that the desorption of an antimicrobial drug from the outer layer of material that fits the wound surface occurs in two directions - to the wound surface due to the difference in concentrations in the wound and the material and in the opposite direction from the outer layer together with absorbed exudate in the inner layer [12]. This process continues until an equilibrium of concentration of the antimicrobial drug in the wound and in the material is established. 
As a result, about $50 \%$ of the antimicrobial drug is desorbed into the wound cavity and on the surrounding skin. A significant part of the drug (about 35\%) remains in the canvas that fits the wound surface, the rest (about 15\%) is concentrated in the inner layer of nonwoven material [13].

In addition to absorbing ability and antimicrobial activity, atraumatic of the material providing a painless change of dressing, preventing damage to the growing epithelium and surrounding skin affects the healing process of postoperative wounds [14].
Atraumatic is characterized by a degree of adhesion to the model of the wound surface which is determined according to GOST 53498 and should not exceed $3 \mathrm{~N} / \mathrm{cm}$. Research conducted in the test center for dressing, suture and polymeric materials of the FSBI "Vishnevsky Institute for Surgery", the results of which are presented in Table 2 , showed that the developed multilayer antimicrobial nonwoven materials have a high level of atraumatic, since the degree of their adhesion to the wound surface model is 2-3 times lower than that of gauze napkins most widely used in medical practice.

Table 2: The degree of adhesion of multilayer antimicrobial nonwoven materials.

\begin{tabular}{|c|c|c|c|}
\hline \multirow{2}{*}{ Antimicrobial Drug } & \multicolumn{3}{|c|}{ The Degree of Adhesion, N/Cm } \\
\cline { 2 - 4 } & Multilayer Antimicrobial Nonwoven Material & Dressing Material “Paul Hartmann” & Gauze Wipes \\
\hline Cefazolin & 2,7 & 2,6 & 5,7 \\
\hline Chlorhexidine digluconate & 1,2 & 2,6 & 5,7 \\
\hline
\end{tabular}

Clinical trial of sterilized dressing with cefazolin and chlorhexidine digluconate treatment were carried out at the Department of Maxillofacial Surgery, Faculty of Dentistry, Yevdokimov Minsk State Medical University of the Ministry of Health of Russia, in the in-patient care of the adult surgical department of the Center for Dentistry and Maxillofacial Surgery and in the FSBI Vishnevsky Institute of Surgery. Medical research included patients with the following diagnoses: maxillofacial abscesses, cellulitis, boils and carbuncles, adenophlegmon and lymphodenitis, infected wounds [15]. Patients who received treatment with gauze dressings were picked out in a separate control group.

The effectiveness of dressing was characterized by a decrease in the microbial contamination of the postoperative wound for 5-7 days of treatment. With use of materials treated with cefazolin, it was $99 \%$, chlorhexidine digluconate-92\%. In the control group during the treatment with use of sterile gauze napkins microbial contamination was reduced by $78 \%$ by day 7 . The results of microbiological research are presented in Table 3.

Table 3: Microbial colonization of postoperative wounds in the dynamics of treatment when using dressing of multilayer antimicrobial nonwoven materials.

\begin{tabular}{|c|c|c|c|c|c|c|}
\hline \multirow{3}{*}{$\begin{array}{l}\text { Biologically Active Package } \\
\text { Treated with Drugs }\end{array}$} & \multirow{3}{*}{$\begin{array}{c}\text { Species of Microorganisms in } \\
\text { Facultative Anaerobic Micro- } \\
\text { flora }\end{array}$} & \multicolumn{4}{|c|}{ Microbial Contamination \% } & \multirow{3}{*}{$\begin{array}{l}\text { The Reduction of } \\
\text { Microbial Contamina- } \\
\text { tion, } \%\end{array}$} \\
\hline & & \multicolumn{2}{|c|}{ At the Opening } & \multicolumn{2}{|c|}{ 5-7 Days of Treatment } & \\
\hline & & $\mathrm{CFU} / \mathrm{ml}$ & $\lg$ CFU & $\mathrm{CFU} / \mathrm{ml}$ & $\lg$ CFU & \\
\hline Cefazolin & $\begin{array}{l}\text { St. aureus - } 30 \% \\
\text { Str. sanguis }-30 \% \\
\text { Ent. faecalis }-40 \%\end{array}$ & $10^{6}$ & $6,0 \pm 0,4$ & $10^{2}$ & $2,0 \pm 0,3$ & 99 \\
\hline Chlorhexidine digluconate & $\begin{array}{c}\text { Enterobacter spp. }-30 \% \\
\text { Str. sanguis -30\% } \\
\text { Ent. faecalis }-20 \%\end{array}$ & $10^{6}$ & $5,9 \pm 0,5$ & $10^{3}$ & $4,0 \pm 0,4$ & 92 \\
\hline Gauze Wipe & $\begin{array}{c}\text { Enterobacter spp. }-30 \% \\
\text { Str. sanguis }-30 \% \\
\text { Ent. faecalis }-30 \%\end{array}$ & $10^{6}$ & $5,9 \pm 0,3$ & $10^{5}$ & $5,1 \pm 0,4$ & 78 \\
\hline
\end{tabular}

The area of postoperative wounds and inflammatory infiltration in the control group was decreased by $7.5 \%$ only by 8 days. The use of dressing with antimicrobial drugs led to a noticeable decrease in the area of infiltration by 4-5 days. That is, with use of the developed dressing, there is the most noticeable healing of the postoperative wound. During the dressing with the use of gauze wipes, most patients had pain syndrome at the time of removal of the dressing, there were noticeable adhesion to the wound surface, trauma of the wound edges accompanied by bleeding. Dressing of multilayer antimicrobial nonwoven materials do not cut blood supply, do not adhere to the wound surface, do not have irritating, allergic, toxic effects. There is a reduction in the period of exudation, the timing of epithelialization compared with gauze dressing [16,17].

Table 4: Characteristics of antimicrobial dressing.

\begin{tabular}{|c|c|c|c|}
\hline $\begin{array}{c}\text { The Composition of the Antimicrobial } \\
\text { Nonwoven Material }\end{array}$ & Antimicrobial Drug & $\begin{array}{l}\text { Maximum Absorption Capaci- } \\
\text { ty, } \mathrm{mg} / \mathrm{Cm}^{2}\end{array}$ & $\begin{array}{l}\text { Antimicrobial Activity (Zone of Growth } \\
\text { Inhibition of Test Microorganisms) }\end{array}$ \\
\hline For the first stage of treatment & Cefazolin & 1092 & $>4,0$ \\
\hline For the second stage of treatment & Cefazolin & 850 & $>4,0$ \\
\hline
\end{tabular}




\begin{tabular}{|c|c|c|c|}
\hline For the third stage of treatment & $\begin{array}{c}\text { Chlorhexidine } \\
\text { digluconate }\end{array}$ & 1139 & $>4,0$ \\
\hline For the fourth stage of treatment & $\begin{array}{c}\text { Chlorhexidine } \\
\text { digluconate }\end{array}$ & 865 & $>4,0$ \\
\hline
\end{tabular}

During the clinical trial, dressing of multilayer antimicrobial nonwoven materials was used in combination with specially designed fixation products, the use of which reduces the time of bandaging of a patient by two to three times. The bandage does not constrain movement, a mimic of facial muscles, can be used by the patient without help. Based on the results of clinical trial, 4 types of dressing were selected, the characteristics of which are presented in Table 4. Recommendations were developed for the use of dressing of multilayer antimicrobial nonwoven materials in surgical departments.

Clinical trial confirmed that at the first stage of treatment with intensive exudation it is necessary to use dressing of multilayer antimicrobial nonwoven materials with a surface density of 500g/ $\mathrm{m}^{2}$ and a thickness of $20.6 \mathrm{~mm}$ (type $\mathrm{A}$ ) with high sorption properties. At the second stage of treatment with moderate exudation, it is necessary to use dressing of multilayer antimicrobial nonwoven materials with a surface density of $300 \mathrm{~g} / \mathrm{m}^{2}$ and a thickness of $10.5 \mathrm{~mm}$ (type B).

To organize the production of multilayer antimicrobial nonwoven materials and dressing made of them, the necessary technological and design documentation was developed. Toxicological tests were carried out and sterilization technology was developed. The developed products received a registration certificate No. RZN 2016/4887, in accordance with which dressing with antimicrobial properties type A will be produced -for postoperative wounds with intensive exudation and type B-for postoperative wounds with moderate exudation.

\section{References}

1. Lopandina SK, Drobyshev AY, Prosacea O, Kozinda ZY, Erofeev 00, et al. (2012) RU Patent for the invention Multi antimicrobial nonwoven material.

2. (2012) Antimicrobial multilayer nonwovens. Medical and technical requirements for development.
3. (2013) Test report of test batches of multilayer antimicrobial nonwovens.

4. IV international conference (2013) Biomaterials and nanobiomaterials: Recent advances and safety and ecology at the Congress Bionanotech 2013. In Heraklion, Crete, Greece.

5. Program and test procedure (ESPA.942417.001 PM).

6. GOST R ISO 14155-2-2014. Clinical studies. Good clinical practice.

7. V international Congress (2014) Biomaterials and nanobiomaterials: The latest achievements in the field of toxicological and environmental safety. In Heraklion, Crete, Greece.

8. Fedorov VD, Chizh IM (2000) Biologically active dressings in the complex treatment of purulent necrotic wounds. Guidelines under the general ed. Acad. Ramsci, Moscow, Russia, p. 38.

9. (2014) Protocol of preclinical testing of multilayer antimicrobial nonwovens and methods of their fixation on the wound surface.

10. Briskin BS (2000) Nosocomial infection and postoperative complications from the standpoint of a surgeon. Infection and antimicrobial therapy 4: 124-128.

11. (2013) Protocol for microbiological testing of antimicrobial multilayer nonwovens. Sbei HPE "Moscow state University of medicine them. In: Evdokimov AI (Ed.), Ministry of healthcare of the Russian Federation, Russia.

12. Vainberg VM, Stegena LM, Illarionov EL, Chufarovsky TI (2003) Biologically active nonwoven fabrics. Technical textiles 6: 24-26.

13. Dobysh SV (1999) Development and study of a new generation of dressings based on modified polymeric materials.

14. Makarova N Ah (2005) Development and research of nonwoven fabrics with antimicrobial properties for medical products. Dissertation abstract, p. 27.

15. Patent RF № 2189210 (2002) Multilayer medical bandage.

16. Prosacea $O$ (2013) The Use of minimized hyperbaric oxygen therapy and antimicrobial dressings in the treatment of patients with phlegmons of the maxillofacial region. Abstract of $\mathrm{PhD}$ thesis.

17. Fokina NA, Gorchakova VM, Note BV, Zasenko N (2006) Antimicrobial non-woven material for bandaging.
Creative Commons Attribution 4.0 International License

For possible submissions Click Here
Submit Article

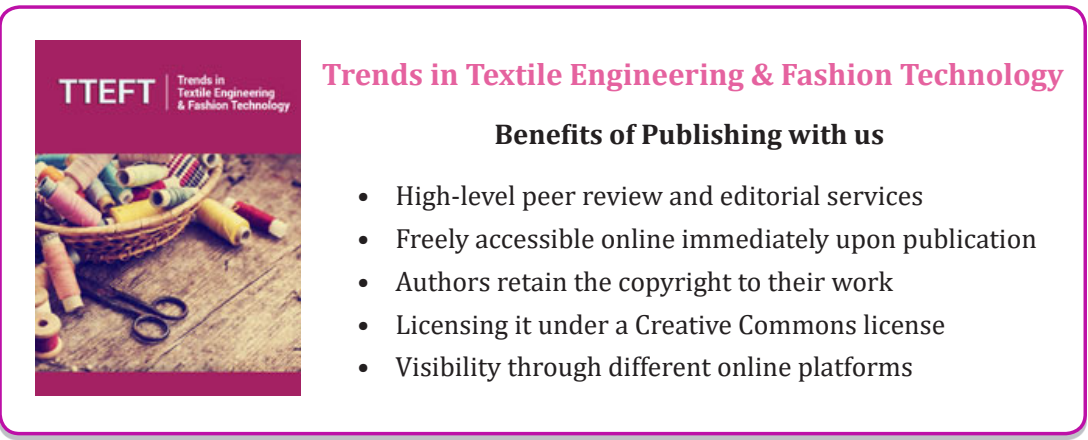

